\title{
Results of the ALICE time-of-flight detector from the 2009 cosmic-ray data taking
}

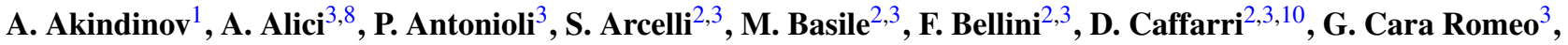

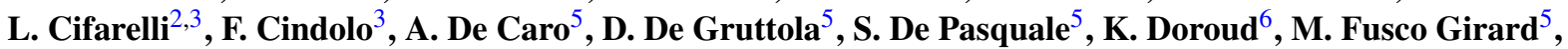 \\ B. Guerzoni ${ }^{2,3}$, D. Hatzifotiadou ${ }^{3}$, W.W. Jung ${ }^{4}$, D.W. Kim ${ }^{4}$, J.S. Kim ${ }^{4}$, S. Kiselev ${ }^{1}$, G. Laurenti ${ }^{3}$, K. Lee ${ }^{4}$, S.C. Lee ${ }^{4}$,

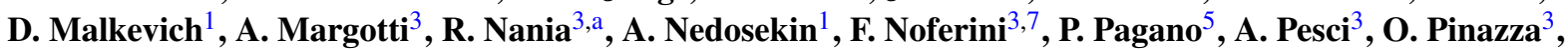 \\ R. Preghenella ${ }^{3,8}$, M. Ryabinin ${ }^{1}$, E. Scapparone ${ }^{3}$, G. Scioli ${ }^{2,3}$, J. Seo ${ }^{6}$, A. Silenzi ${ }^{2,3}$, K. Voloshin ${ }^{1}$, M.C.S. Williams ${ }^{3}$, \\ C. Zampolli ${ }^{7,9}$, A. Zichichi ${ }^{2,3,8}$ \\ ${ }^{1}$ Institute for Theoretical and Experimental Physics, Moscow, Russia ${ }^{\mathrm{b}}$ \\ ${ }^{2}$ Dipartimento di Fisica dell'Università, Bologna, Italy \\ ${ }^{3}$ Sezione INFN, Bologna, Italy \\ ${ }^{4}$ Department of Physics, Gangneung-Wonju National University, Gangneung, Republic of Korea \\ ${ }^{5}$ Dipartimento di Fisica dell'Università and INFN, Salerno, Italy \\ ${ }^{6}$ World Laboratory, Geneva, Switzerland \\ ${ }^{7}$ INFN-CNAF, Bologna, Italy \\ ${ }^{8}$ Museo Storico della Fisica e Centro Studi e Ricerche "Enrico Fermi", Roma, Italy \\ ${ }^{9}$ CERN, Geneva, Switzerland \\ ${ }^{10}$ Present address: INFN and University of Padova, Padova, Italy
}

Received: 8 March 2010 / Revised: 25 April 2010 / Published online: 29 June 2010

(C) The Author(s) 2010. This article is published with open access at Springerlink.com

\begin{abstract}
The Time-Of-Flight detector (TOF) of the ALICE experiment at the CERN LHC is based on Multi-gap Resistive Plate Chambers (MRPCs). The TOF detector consists of 152928 readout channels covering a total area of $141 \mathrm{~m}^{2}$. In this paper the results of the calibration with cosmic-ray data collected during 2009 are presented.
\end{abstract}

\section{Introduction}

The ALICE experiment will study heavy ion collisions at high energy at the CERN LHC [1-3].

The Time-Of-Flight (TOF) detector [4] in ALICE is made of 1593 Multi-gap Resistive Plate Chambers (MRPCs) [5]. The detector is subdivided into 18 azimuthal sectors, each one containing a SuperModule (SM) more than $9 \mathrm{~m}$ long. Each SM contains 91 MRPCs distributed into 5 gas-tight modules. ${ }^{1}$ A total of 152928 readout pads (each $2.5 \times 3.5 \mathrm{~cm}^{2}$ ) cover an area of $141 \mathrm{~m}^{2}$.

\footnotetext{
${ }^{1}$ Three SMs have been installed without the central module in order to reduce the amount of material in front of the high resolution electromagnetic calorimeter (PHOS).
}

a e-mail: nania@bo.infn.it

${ }^{b}$ RFBR grant 08-02-91911-CERNa.
The TOF design goal was to reach a global time resolution of about $100 \mathrm{ps}$ in order to identify pions and kaons up to $2.5 \mathrm{GeV} / c$ and protons up to $4 \mathrm{GeV} / c$ in the pseudorapidity interval $[-0.9,+0.9]$ and full azimuthal coverage [6].

In this paper the TOF performance is described, as measured with cosmic rays during a data taking period which lasted from August to November 2009. During this period the TOF took data for more than 1300 hours and collected more than 200 million events. In addition, the TOF had the very important role of providing the triggers for the other ALICE detectors.

Test beam results may be found in [7] and [8], where the time resolution of the MRPCs has been measured to be bet-

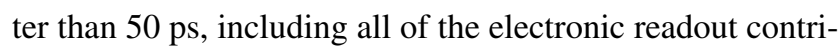
bution. The TOF construction and detector assembly are described in detail in [8], while the commissioning work may be found in [9], together with preliminary results with cosmic rays from the 2008 data taking.

\section{TOF cosmic trigger}

The TOF trigger is constructed from 1728 trigger channels, each covering $888 \mathrm{~cm}^{2}$ (equivalent to the area of one MRPC). This information is processed and sent within $<800 \mathrm{~ns}$ to the ALICE Central Trigger Processor (CTP) 
which provides the level zero trigger (L0) for the experiment. Further details on the TOF trigger system can be found in [10].

Contrary to the Physics Report conclusions [1-3], where triggering on single muons was considered unfeasible, during the TOF commissioning in 2008 it came out that the MRPC dark noise was strongly reduced with respect to the measurements taken with prototypes $[9,10]$. This opened the possibility to search for various cosmic-muon topologies and during the 2009 cosmic-ray run the TOF detector provided several triggers, selecting single- and multiple-track events in the whole detector and/or in specific detector regions.

The ALICE Collaboration took benefit of two main TOF triggers, named $0 \mathrm{OCP}$ and $0 \mathrm{OB} 3$, and of two dedicated triggers, named 0OIT and 0OBE. Both the 0OCP and 0OB3 triggers were used as global triggers to select data for the general ALICE data acquisition with several detectors.

An event is selected by 0OCP if at least four channels have fired anywhere in the TOF. The 0OCP trigger, with a stable rate around $10 \mathrm{~Hz}$, was mainly used as a trigger for cosmic-ray physics, being well suited for multiple muons event selection.

As far as 0OB3 is concerned, this is based on a simple event topology (Fig. 1): a trigger is delivered when a SM has fired in coincidence with another SM, back-to-back on the azimuthal plane. To enlarge the acceptance for lowmomentum muons, this condition is relaxed allowing the trigger to fire also if just one of the three SMs on either side of the back one has fired (three is reduced to one for S00 and S08 configurations). The rate of such a trigger was stable around $100 \mathrm{~Hz}$.

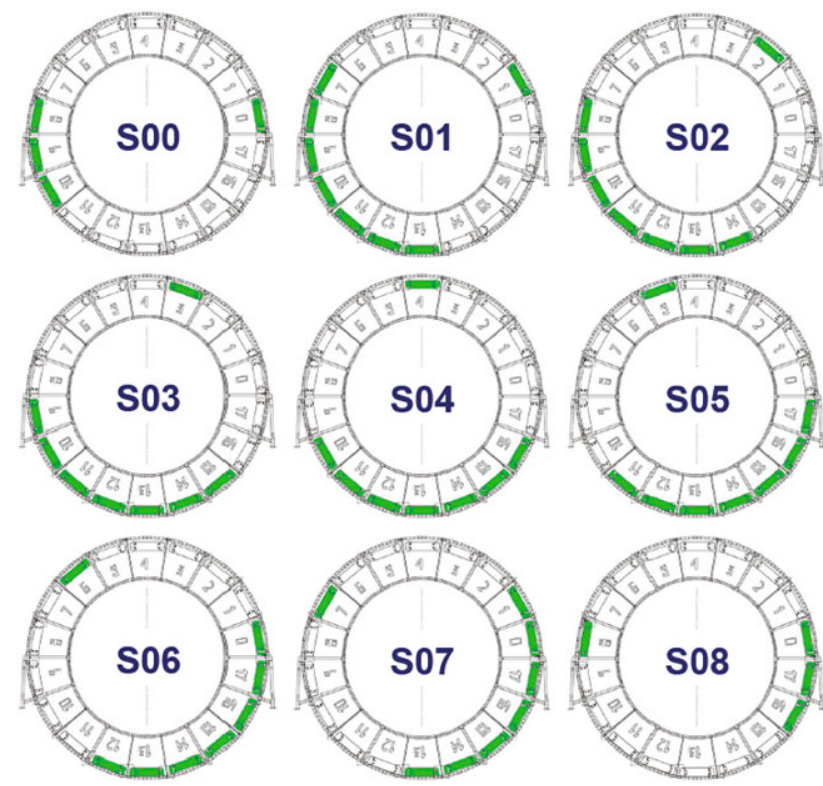

Fig. 1 The TOF sector coincidences for the 0OB3 trigger configuration
The main goal of the $0 \mathrm{OB} 3$ trigger was the selection of a large sample of cosmic muons to be used for tracking detector studies. Its successful performance allowed to collect hundreds of million muons. The trigger purity may be estimated measuring the in-gate and out-of-gate trigger rates. ${ }^{2}$ Figure 2 shows the result as well as the difference between the two, which corresponds to the true muon rate and amounts to about $45 \%$ of the trigger sample.

As an alternative evaluation of the trigger purity, the quantity $\Delta t-L / c$ is computed, where $\Delta t=t-t_{0}$ is the time-of-flight of the candidate particle between the two TOF hits in opposite sectors and $L / c$ is the expected time-offlight evaluated from the distance $L$ of the hit points on the TOF assuming ultra-relativistic muons, with $c$ the velocity of $\operatorname{light}^{3}$ (Fig. 3). Figure 4 shows the distribution of $\Delta t-L / c$. At this stage no tracking information is used and no TOF detector calibration is applied, while all the triggered events with two TOF hits on opposite sectors are included. A clear peak is observed around zero corresponding to muon candidates. This peak accounts for more than $40 \%$ of the total triggers and its width gives for the TOF a raw time resolution of about $1 \mathrm{~ns}$.

The quality of this trigger was also checked looking at tracks in the ALICE Time Projection Chamber (TPC) [11, 12]. However the TPC length along the beam axis $(5 \mathrm{~m})$ is shorter than the TOF one $(7.4 \mathrm{~m})$ and this results in a smaller

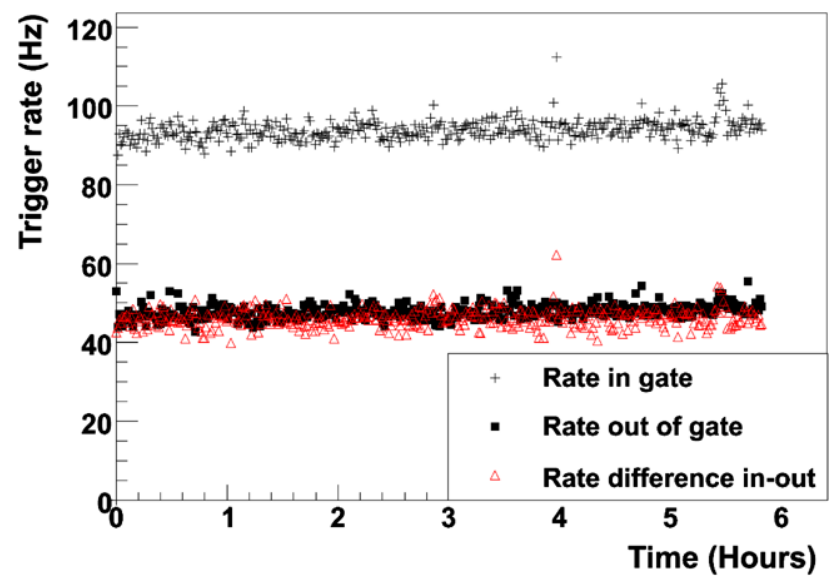

Fig. 2 The measured rate of in-gate and out-of-gate coincidences and their difference (true muon candidates)

\footnotetext{
${ }^{2}$ Hereafter we define as "gate" the time interval between two consecutive positive ALICE clock transitions, i.e. $25 \mathrm{~ns}$. The "in-gate" are coincidences between up and down TOF sectors taking properly into account the time-of-flight for down-going muons; in the out-of-gate triggers one signal is delayed by $250 \mathrm{~ns}$ to evaluate accidental coincidences due the MRPC random noise which amounts to about 800 $\mathrm{Hz} / \mathrm{m}^{2}[10]$.

${ }^{3}$ The expected most probable value for cosmic muons reaching the ALICE detector is above $10 \mathrm{GeV} / \mathrm{c}$ and the fraction below $1 \mathrm{GeV} / \mathrm{c}$ is negligible.
} 


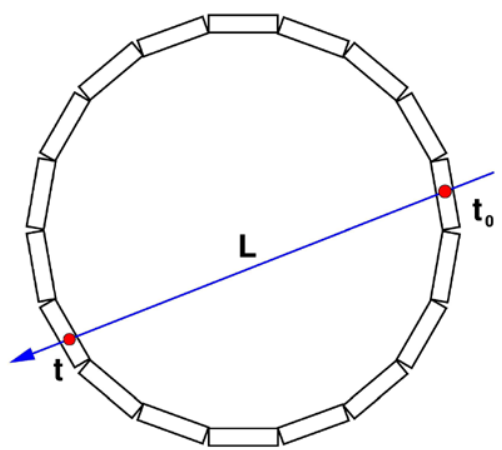

Fig. 3 Schematic drawing of a cosmic-ray track hitting twice the TOF array

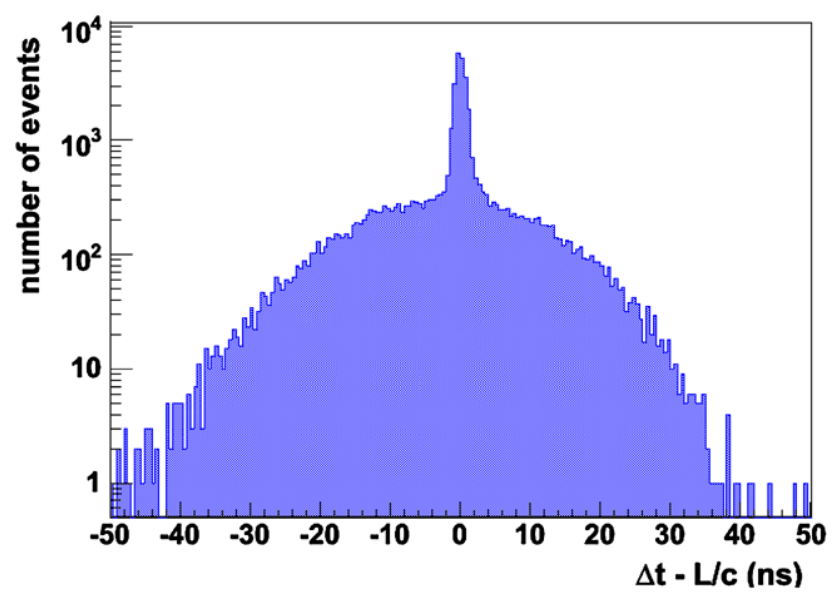

Fig. 4 Distribution of $\Delta t-L / c$ for events selected by the 0OB3 trigger. Uncorrected time measurements are used

fraction of reconstructed tracks with respect to the intrinsic purity of the trigger. Nevertheless this trigger provided several millions of tracks well suited for the determination of the TPC calibration parameters.

The arrival direction of the muons selected in the peak of Fig. 4 was also studied: Fig. 5 reports the $\phi-\theta$ correlation, with vertical muons at $\theta=90^{\circ}$ and $\phi=270^{\circ}$, with $\phi$ calculated from the horizontal axis ${ }^{4}$ with respect to the direction of the down-going muon. A clear excess is observed at $\theta=120^{\circ}$ corresponding to the access shaft of the ALICE experimental hall.

The TOF delivered two more dedicated triggers: 0OIT and 0OBE. The first one is used to trigger the ALICE Inner Tracking System (ITS) detectors. The 0OBE trigger is used to trigger the ALICE Electromagnetic Calorimeter (EM$\mathrm{CAL}$ ). Both $0 \mathrm{OIT}$ and $0 \mathrm{OBE}$ are subsets of the $0 \mathrm{OB} 3$ trigger.

${ }^{4}$ In the ALICE reference frame the horizontal axis points to the inside of the LHC beam ring, while the $z$ axis is along the beam direction.
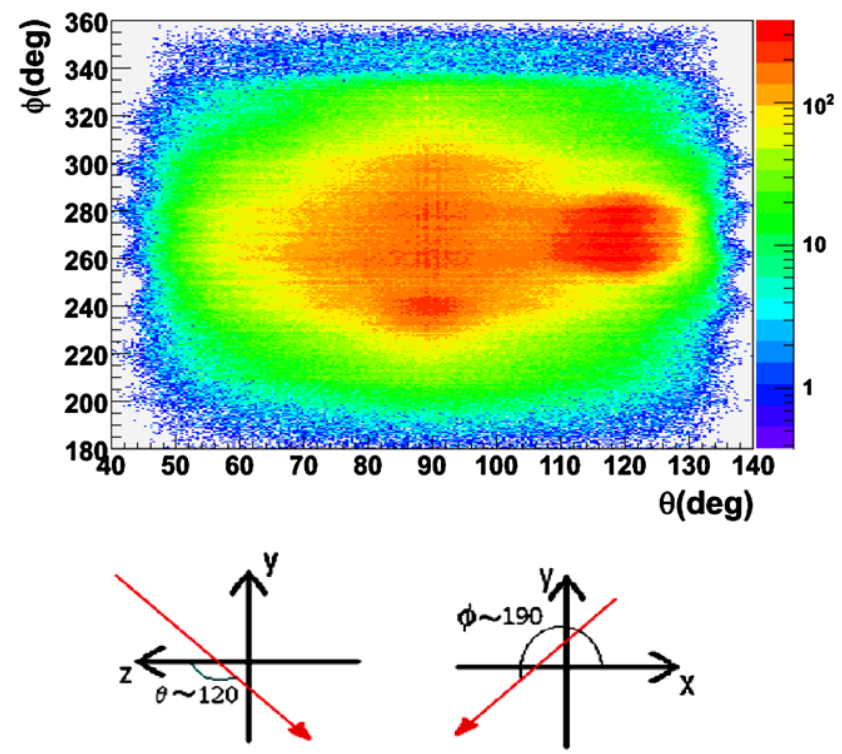

Fig. 5 (Top) $\phi-\theta$ correlation for the muons triggered by the TOF. (Bottom) Schematic definition of the $\phi$ and $\theta$ angles in the ALICE reference system

\section{Cosmic-ray data sample and event selection}

The following studies have been performed with a subset of the cosmic-ray data collected by ALICE during 2009 without magnetic field. The selected runs, recorded between mid-September and mid-October, have been chosen among the reconstructed ones with the requirement of having good calibration parameters for the TPC thus enabling accurate track reconstruction in the central barrel.

The event selection is performed on the basis of the topology requiring just one muon reconstructed by the TPC. This selection is imposed by the need to reduce possible backgrounds and wrong matches due to cosmic muons interacting with the ALICE magnet or other detectors. A cosmic ray traversing the ALICE central barrel is not reconstructed as a single track, but as two separate tracks emerging from a common point, here called muon vertex, and directed outwards (Fig. 6). For this reason, a single-muon
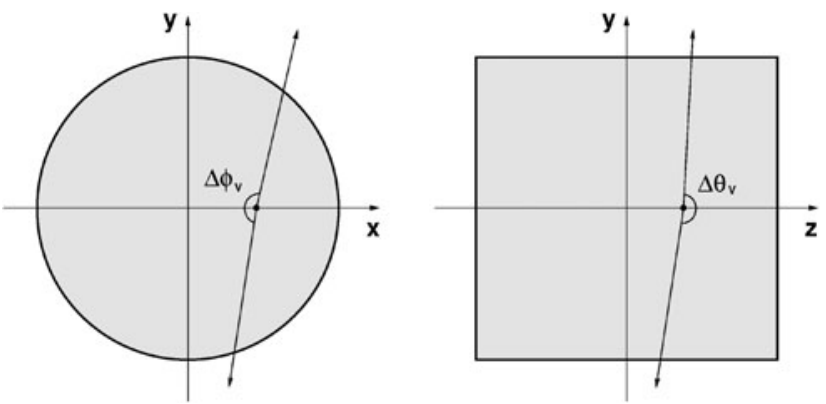

Fig. 6 Schematic drawing of a cosmic ray reconstructed as two tracks within the ALICE reference frame. The angles $\Delta \theta_{\mathrm{v}}$ and $\Delta \phi_{\mathrm{v}}$ defined for track quality cuts are also shown 
event is tagged with the requirement of having exactly two TPC-reconstructed tracks pointing towards opposite directions. Further quality cuts are applied to guarantee the best cosmic-ray track reconstruction on the basis of the track parameters at the muon vertex. Let us call $\Delta x_{\mathrm{v}}, \Delta y_{\mathrm{v}}, \Delta z_{\mathrm{v}}$ the spatial distance between the tracks in the $x, y, z$ directions, respectively, at the muon vertex and $\Delta \phi_{\mathrm{v}}, \Delta \theta_{\mathrm{v}}$ the difference between the direction of the tracks in the $x y, z y$ planes, respectively (Fig. 6); the applied selection cuts are:

- $\left|\Delta x_{\mathrm{v}}\right|<1 \mathrm{~cm},\left|\Delta y_{\mathrm{v}}\right|<1 \mathrm{~cm}$ and $\left|\Delta z_{\mathrm{v}}\right|<1 \mathrm{~cm}$;

- $\left|\Delta \phi_{\mathrm{v}}-\pi\right|<10 \mathrm{mrad}$

- $\left|\Delta \theta_{\mathrm{v}}-\pi\right|<7 \mathrm{mrad}$.

As a final selection requirement, both tracks belonging to the single muon must have been matched with a signal of the TOF detector, thus enabling the measurement of the muon time-of-flight and the distance between the two hit points on the TOF array. After the above cuts about 1.7 million singlemuon events are selected.

\section{Track-matching performance}

The good calibration of the TPC detector allows to reconstruct the track parameters with accuracy, thus enabling a good determination of the track position at the TOF layer. Thanks to this precise information an alignment of the TOF detector with respect to the TPC was possible. The procedure, not documented in this paper, measures the actual position of TOF SuperModules by minimizing the residuals between the TPC track position extrapolated at the TOF layer and the TOF-matched hit. The output, three shifts and three rotation angles for each sector, is then used to correct the geometrical description of the apparatus.

After this alignment, the distance between the track hit point and the pad center in the two directions $x, z$ of the pad reference frame (Fig. 7), namely $\Delta x_{\text {pad }}$ and $\Delta z_{\text {pad }}$ respectively, gives an estimate of the track-match quality. The

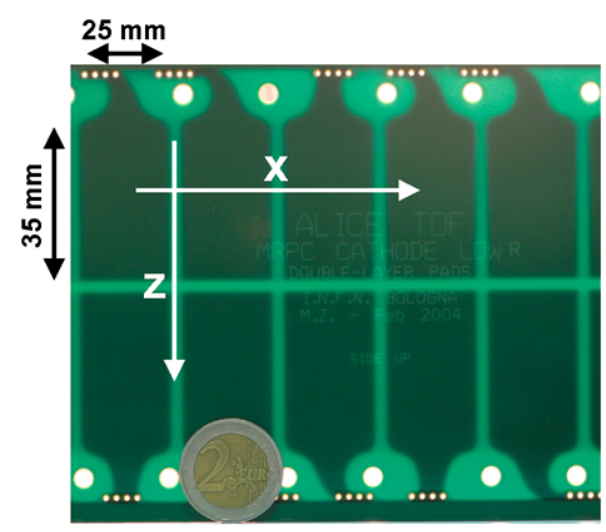

Fig. 7 Printed circuit board containing the TOF readout pads. Superimposed are the pad reference frame and the pad dimensions results are summarized in Fig. 8. The full width at half maximum of the distributions is in accordance with the size of the TOF pad and the smooth edges indicate a track-point resolution at the TOF of about $4 \mathrm{~mm}$.

\section{Timing performance}

The time resolution of the TOF detector has been estimated by using again the quantity $\Delta t-L / c$, but now with calibrated time measurements and TPC track information.

A preliminary result has been already obtained with the cosmic-ray data collected during 2008. Despite a limited statistics of about 10000 muon tracks, a measured single-hit time resolution of $130 \mathrm{ps}$ could be reached for the fraction of TOF sectors then in operation. This result is documented in [9] together with the details about the calibration procedure implemented with the cosmic rays and used again to obtain the results presented herein.

As a first step the TOF calibration was repeated with a portion of the 2009 data, profiting of the increased statistics and extending the analysis to the whole TOF. No cuts on the quality of the TPC tracks were applied and the matching distance between the extrapolated track and the associated TOF pad center was required to be less than $10 \mathrm{~cm}$, without any specific alignment. Figure 9 shows the result. An average resolution of $\sigma_{\Delta t}=157 \mathrm{ps}$ is obtained. The timing performance of the TOF detector, that is the single-hit time resolution, is then evaluated taking into account that time-of-flight measurements are done via two independent time measurements. The single-hit resolution is therefore $\sigma_{t}=\sigma_{\Delta t} / \sqrt{2}$, which leads to a global TOF time resolution of $110 \mathrm{ps}$ over the whole detector, improved with respect to the 2008 result.

However, the quite large number of muon events selected from the 2009 cosmic-ray data allowed to apply more severe cuts to investigate the detector performance limit. Using the previously described track quality cuts and improved alignment, it was possible to compute with greater accuracy the length traveled by the cosmic ray between the hit points on the TOF and to reject tracks too far from the associated TOF hit thus indicating poor track reconstructed parameters. Moreover the extrapolated track was requested to be within the associated TOF pad to reduce the probability of having tracks crossing the TOF in between two pads and causing two neighboring readout channels to fire, which worsened the single-pad time resolution as observed in previous tests [7]. Defining as $\Delta x_{\text {pad }}$ and $\Delta z_{\text {pad }}$ the distance between the track hit point and the pad center in the $x$ and $z$ coordinates of the pad reference frame (Fig. 7) respectively, the following cuts were applied:

- $\left|\Delta x_{\text {pad }}\right|<1.25 \mathrm{~cm}$;

- $\left|\Delta z_{\text {pad }}\right|<1.75 \mathrm{~cm}$. 
Fig. 8 Reconstructed hit point on TOF readout pads with respect to the pad center (top). Distribution of the

track-matching residuals in the bending plane (middle) and in the longitudinal direction (bottom). The vertical lines define the size of the readout pad and the cuts applied for time resolution measurement
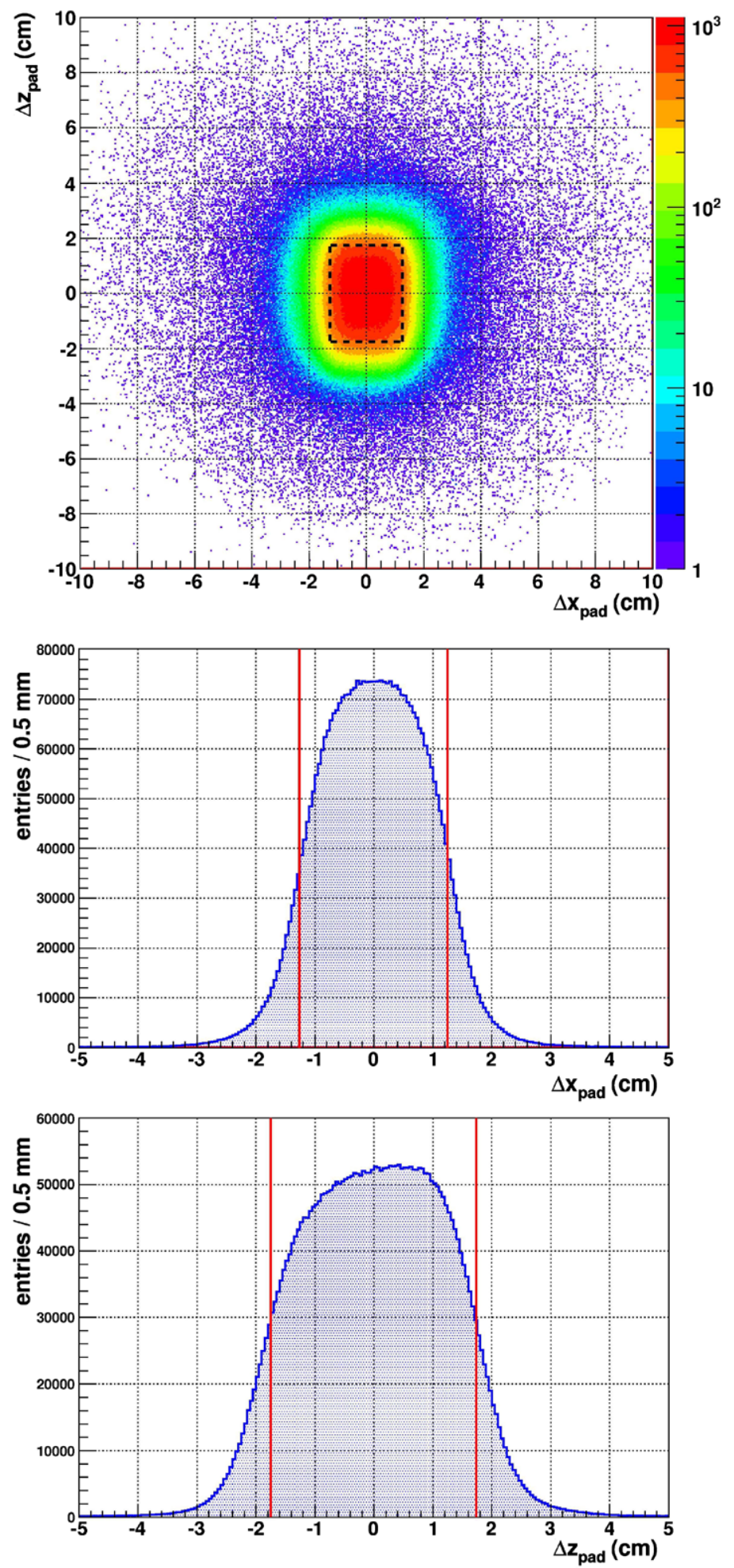

These requirements force the hit point to be inside the pad and each one rejects about $15 \%$ of the hits (Fig. 8). In the present analysis no correction was attempted for the position of the hit inside the pad (time walk effect).
The channel-by-channel time slewing corrections, measurable thanks to the Time-Over-Threshold information ${ }^{5}$

${ }^{5}$ The Time-Over-Threshold (TOT) circuit provides for each readout pad of the MRPCs a time width fairly proportional to the pulse height (i.e. charge) of the signal [13]. 
stored in the data, are still not possible with the present statistics. This correction is thus performed only at a global level. However, in this attempt to study the best TOF performance, Time-Over-Threshold values below $11.0 \mathrm{~ns}$ or greater than $16.0 \mathrm{~ns}$ are excluded because the time slewing

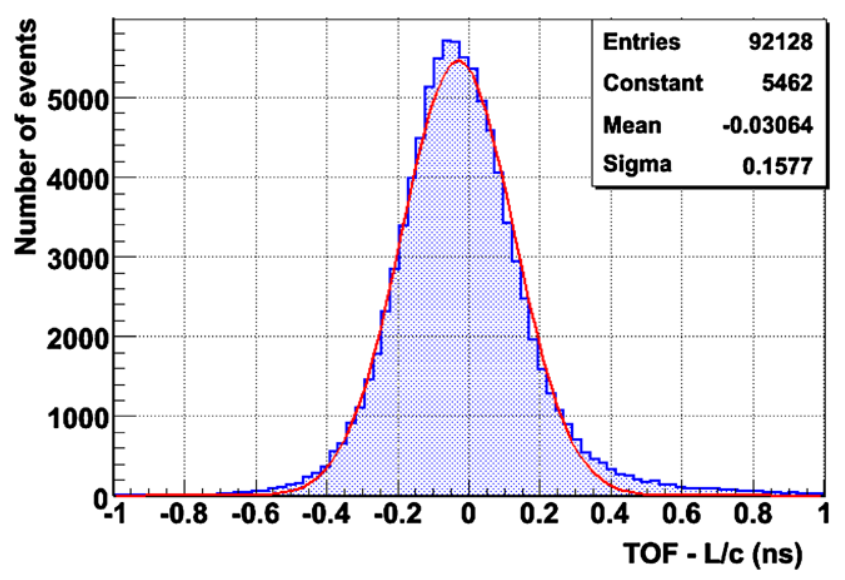

Fig. 9 Muon time-of-flight distribution obtained after TOF calibrations corrections have been found to strongly depend on each pad. This cut rejects another $6 \%$ of the TOF hits (Fig. 10).

The selection of good TOF-matched hits reduces the final sample of cosmic muons to about $45 \%$ of the one obtained after the cuts on the TPC track quality. The distribution of the residual time-of-flight $\Delta t-L / c$ is shown in Fig. 11. An average resolution of $\sigma_{\Delta t}=125 \mathrm{ps}$ is obtained, corresponding to a single-hit of $\sigma_{t}=88 \mathrm{ps}$ which represents the present limit of the TOF time resolution.

\section{Conclusions}

During the period from August to November 2009 the TOF collected few hundred millions of cosmic-ray data running with no major problems and providing the main source of trigger for the ALICE detector calibration.

The data showed a good match between the TOF and the main ALICE tracking detectors. An excellent time resolution of 110 ps was achieved. Applying stronger cuts on the quality of the track reconstruction and on the TOF matching, the TOF time resolution reached 88 ps, with still a good
Fig. 10 (Top)

Time-Over-Threshold (TOT) distribution of the hits associated with the cosmic-ray track. The vertical lines define the applied cuts for the time resolution measurement. The small shoulder on the right is related to rescattering of the electronics with big signals. (Bottom) Correlation between measured time and TOT (providing the time slewing correction), integrated over all channels: the black line indicates the average value for each bin (profile plot)
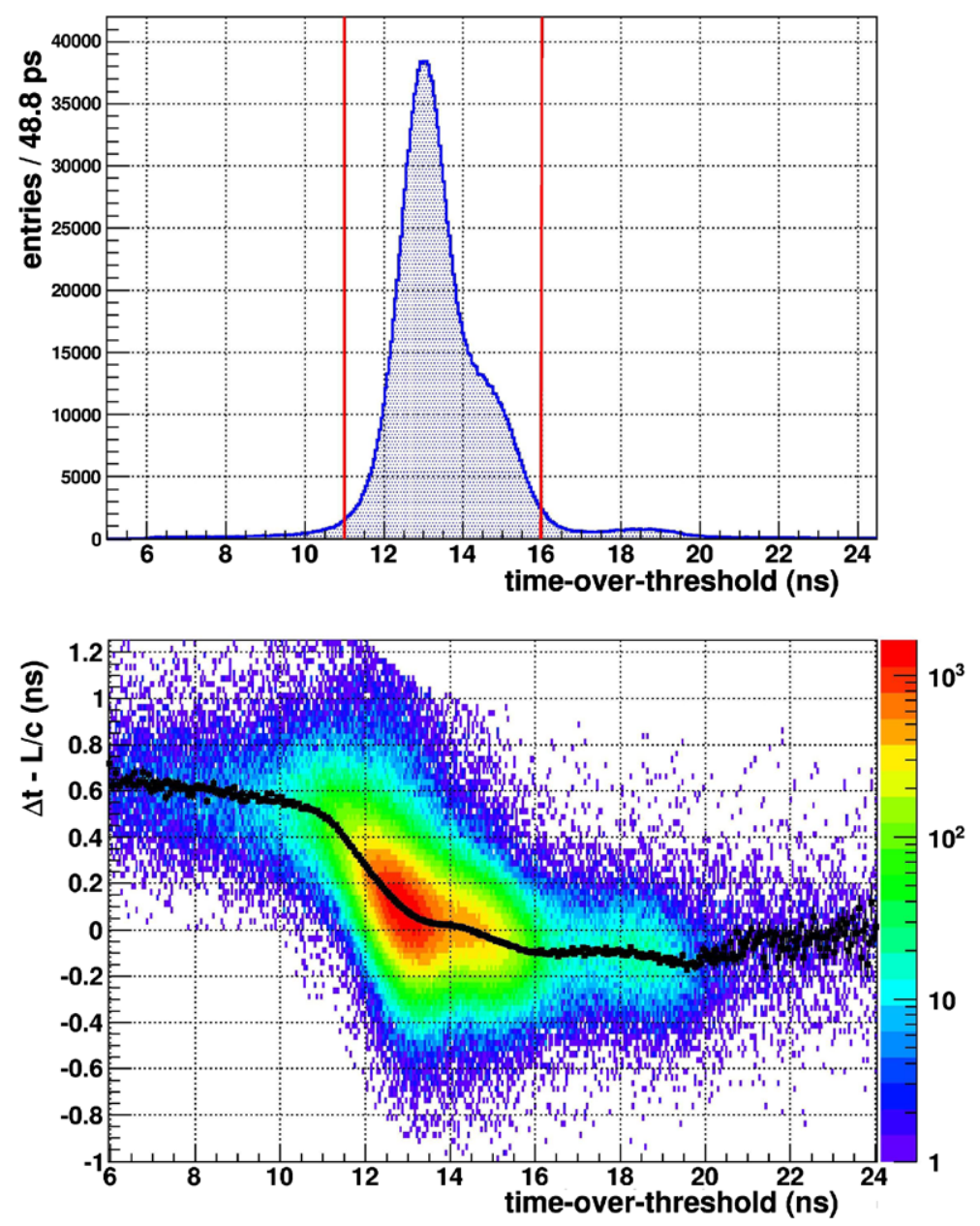


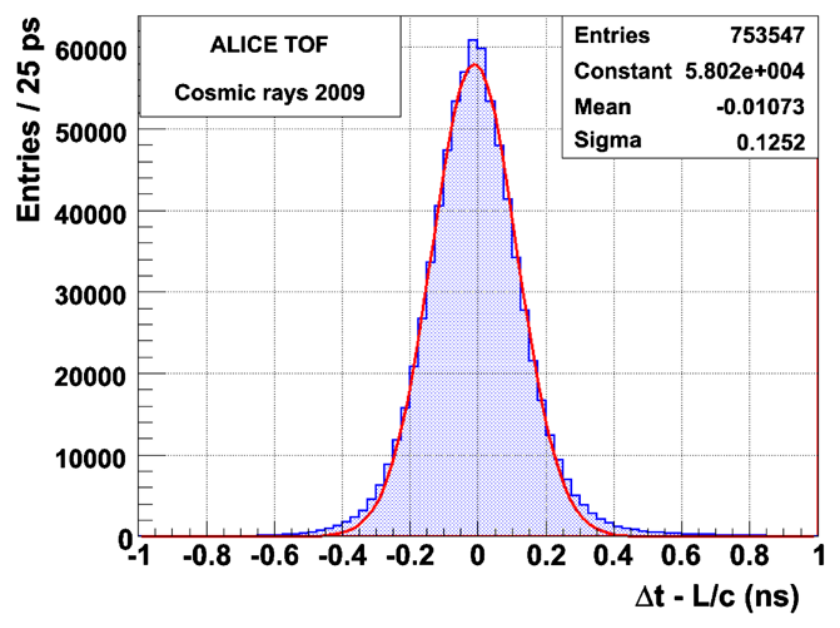

Fig. 11 Muon time-of-flight distribution obtained after TOF calibrations and more stringent requirements on tracking and TOF matching

margin for improvement expected from channel-by-channel time slewing and from precise time walk corrections for each readout pad.

Acknowledgements We thank all colleagues from the ALICE Collaboration who made possible the analysis of the cosmic data reported in the present paper. The ALICE Management, the Technical and Run Coordination, the DAQ, DCS, ECS and Trigger Groups are especially acknowledged for their help and support during the data taking period.
Open Access This article is distributed under the terms of the Creative Commons Attribution Noncommercial License which permits any noncommercial use, distribution, and reproduction in any medium, provided the original author(s) and source are credited.

\section{References}

1. ALICE Collaboration, J. Phys. G: Nucl. Part. Phys. 30, 1517 (2004)

2. ALICE Collaboration, J. Phys. G: Nucl. Part. Phys. 32, 1295 (2006)

3. ALICE Collaboration, J. Instrum. 3, S08002 (2008)

4. ALICE Collaboration, Addendum to TOF Technical Design Report CERN/LHCC 2002-016

5. E. Cerron-Zeballos et al., Nucl. Instrum. Methods A 374, 132 (1996)

6. A. Akindinov et al, Eur. Phys. J. C 32, S02, 165 (2004)

7. A. Akindinov et al., Nucl. Instrum. Methods A 532, 611 (2004)

8. A. Akindinov et al., Nuovo Cim. B 124, 235 (2009)

9. A. Akindinov et al., Nucl. Instrum. Methods A 615, 37 (2010)

10. A. Akindinov et al., Nucl. Instrum. Methods A 602, 372 (2009)

11. ALICE Collaboration, ALICE TPC Technical Design Report, CERN/LHCC 2000-001

12. The TPC Collaboration, http://www-library.desy.de/cgi-bin/ spiface/find/hep/wwwauthors?key=8521492, Nucl. Instrum. Methods A. to be published

13. F. Anghinolfi et al., Nucl. Instrum. Methods A 533, 183 (2004) 